\title{
Managing the adverse events of intravesical bacillus Calmette-Guérin therapy
}

This article was published in the following Dove Press journal:

Research and Reports in Urology

23 October 2015

Number of times this article has been viewed

\section{Karel Decaestecker Willem Oosterlinck}

Department of Urology, Ghent University Hospital, Ghent, Belgium

Correspondence: Willem Oosterlinck Department of Urology, Ghent University Hospital, De Pintelaan I85, 9000 Ghent, Belgium

Tel +32472256 I 20

Fax +32 93323889

Email willem.oosterlinck@ugent.be
Abstract: This paper provides recommendations on the management of complications arising from intravesical treatment with bacillus Calmette-Guérin (BCG) for nonmuscle-invasive bladder tumors. There is minimal recommendations currently available as randomized trials on the side effects of intravesical BCG are lacking and severe complications are usually described in case reports only. All physicians giving intravesical BCG should be aware of the possible complications that could arise and how to treat these. The incidence of bladder irritation, general malaise, and fever is very high, while severe complications remain rare. Approximately $8 \%$ of patients have to stop treatment because of these complications. BCG infections and reactions can occur anywhere in the body, and may happen straight away or even several months or years after BCG treatment, making early diagnosis difficult. Additionally, correct diagnosis is hampered by the uncertain appearance of BCG in tissue and body fluid. An essential step in the management complications arising from BCG is written information for both the family doctor and the patient on the possible adverse events and their management. Recent data demonstrated that none of the earlier advocated methods to prevent BCG toxicity are valid: lowering the dose, tuberculostatic drugs, or oxybutynin. Severe complications are treated with three or four tuberculostatics over 3-12 months, depending on the severity of the situation. Corticosteroids are an essential therapy in BCG septicemia. Nonsteroidal anti-inflammatory drugs and corticosteroids can manage efficiently the immunological complications.

Keywords: BCG, intravesical therapy, complications

\section{Introduction}

Bacillus Calmette-Guérin (BCG) has been used to treat nonmuscle-invasive bladder tumors for $>40$ years. It is one of the most successful biotherapies for cancer in use. Despite this long clinical experience, the mechanism of its therapeutic effect is still under investigation. Available evidence suggest that urothelial cells, including the bladder cancer cells themselves, cells of the immune system, and their secretions both have crucial roles in its antitumor effect. ${ }^{1}$ Several of the side effects are related to this strong immune response and others to the fact that living bacteria are instillated in the bladder.

$\mathrm{BCG}$ is found to be the most effective prophylactic treatment for patients with high-risk nonmuscle-invasive urothelial bladder neoplasms, and its use is therefore recommended in international guidelines. ${ }^{2}$ In intermediate risk disease, it was also found more effective than intravesical chemotherapy but its use should be outweighed against its potential severe toxicity. BCG has no place in the treatment of low-grade urothelial neoplasms except in the rare cases where chemotherapy is unable to reduce the recurrence rate of the tumors. ${ }^{3}$ The severe side effects are a major reason to avoid 
the use of BCG. Nevertheless, BCG remains an important and common treatment to avoid the need for cystectomy in urothelial cancer. Every physician treating this disease should therefore know how to prevent and manage the complications of BCG treatment.

\section{Previous reviews on management of BCG complications}

A committee of international experts in bladder cancer management, known as the International Bladder Cancer Group, published practical recommendations for prevention and treatment of intravesical BCG adverse events in October 2008. ${ }^{4}$ This was based on a review of the literature and to a large extent on expert opinion because literature did not deliver a level of evidence greater than level 3. As several of the authors of this article were also involved in previous reviews, ${ }^{5-8}$ this document reflects the opinions that were already published before 2008. Some of their recommendations did not survive. After 2008, prevention of adverse events with ofloxacin, lowering BCG dose, and use of oxybutynin were not confirmed to be effective in randomized trials (see below).

To our knowledge and after a PubMed search from January 2000 to May 2015 with the terms "BCG complications", the current article is the last complete review with treatment recommendations published.

\section{Frequency and timing of the side effects}

The largest and most recent published study with $\mathrm{BCG}$, including 1,316 patients, is the EORTC study comparing one-third dose with full dose BCG and 1 year with 3 years of maintenance BCG. ${ }^{9,10}$ It reports the side effects in a standard way, and also a recommendation on the treatment of the complication was provided in the study protocol. Its publication on the side effects ${ }^{10}$ contains interesting and recent information on BCG toxicity. Among the 1,316 patients who started BCG, $62.8 \%$ reported local side effects and $30.6 \%$ had some form of systemic side effects, from a frequent short period of fever and general malaise to rare severe systemic complications. This is in line with the previous reports on this subject and illustrates the high frequency of the side effects. ${ }^{6}$

The most frequent local side effects in the EORTC study ${ }^{9}$ are complaints of BCG-induced cystitis (35\%) and bacterial infection $(23.3 \%)$, frequency of more than once per hour in $23.6 \%$, and macroscopic hematuria in $22.6 \%$. The most frequent systemic side effects were general malaise in $15.5 \%$ and fever in $8.1 \%$. BCG sepsis was observed in four patients $(0.3 \%)$. In contrast to the general belief that side effects increase over time, frequency was similar in the induction course (first six instillations), during the first year, and in the 2 following years. Most treatment discontinuations for severe side effects occurred in the first year $(6.2 \%$ on a total of $7.8 \%$ discontinuations over the whole treatment period). Severe reactions can already appear at the first instillation, ${ }^{11}$ but obviously the side effects are not dependent on the number of instillations but upon the host. These results confirm the observation of a previous EORTC study (30911) where the majority of the side effects occurred within the first year, ${ }^{12}$ and still $19 \%$ of the patients stopped treatment because of toxicity. Obviously, the discontinuation rate diminished with increasing experience among urologists with BCG as only $7.8 \%$ stopped treatment in the last study.

\section{Type of severe side effects}

Severe side effects continue to be reported in the literature. BCG infection or immune reactions can appear wherever in the body, in any organ, with very unusual clinical manifestations in different medical specialties. A review on the subject was published in August $2014^{13}$ and gives an extensive list of described severe complications: prostatitis, orchiepididymitis, balanitis, osteomyelitis, and acute infectious arthritis at different places in the skeleton, infected orthopedic and vascular prostheses, rheumatological complications, mycobacterial pneumonia, interstitial pneumonitis due to hypersensitivity, hepatitis, nephritis, vasculitis, mycotic aneurisms, disseminated BCG and septicemia, and finally the local complication of ureteral obstruction and bladder contracture. From June 2014 to May 2015, we found another ten reports on severe complications. ${ }^{14-23}$ So the things that can happen are very rare but quite impressive and in any organ of the body.

\section{Difficulties in diagnosis of the severe side effects}

If the side effects occur shortly after the instillation, the relationship with BCG can be made easily. Nevertheless, the side effects mostly occur when the patient is already back at home and generalists are not familiar with the complications of BCG. Therefore, it is highly advocated to inform the family doctor of the patient on the treatment and its possible complications, alarm symptoms, and practical attitude to solve the problem.

A second problem is that complications occur late, up to years after the treatment, when the urologist, who gives it, is no longer involved. Additionally, many of the complications 
give symptoms elsewhere in the body, and the patient goes to specialists who, again, are unfamiliar with BCG manifestations or even unaware of this treatment. Therefore, an information document should be delivered to each patient treated with the possible late complications. This is the way we handle it since many years. Although we have no proof, we are convinced that this can help to come to a more rapid diagnosis by colleagues of other specialties. This is important as the outcome of a complication is often dependent on early treatment initiation.

Even if a BCG complication is suspected, it may be difficult to prove it. Acid-fast staining, culture, and polymerase chain reaction testing are often negative. Tissue biopsies and cultures should be performed to evaluate noncaseating granuloma formation and the presence of Mycobacterium bovis, ${ }^{24}$ realizing, however, that this has a low yield. As a result, a high clinical suspicion is critical in order to prevent delays in treatment initiation. ${ }^{22}$

\section{Differences in adverse events among different BCG strains}

Side effects seem similar for all BCG strains used (Tice, Connaught, Pasteur, RIVM), but frequency differs from one study to another. No differences between strains in side effects have been noted in a meta-analysis ${ }^{25}$ and in a recent study comparing Tice versus Connaught strain. ${ }^{26}$ The dose and treatment schedules used differ among studies, and therefore, comparison among them is difficult. Ideally, the dose should be expressed on colony-forming units as there are strong variations of factors $1-4$ among the commercial preparations. Many studies do not mention this information and express the dose in milligrams.

Another problem is that the side effects of BCG have not been evaluated in a standard way in many studies, resulting in large differences in the frequency of local side effects of BCG. A classification of these side effects, taking into account severity and duration, has been proposed and validated, ${ }^{7}$ but this was not commonly applied by the urologic community.

\section{Prevention strategies to diminish toxicity Avoid factors favoring side effects}

Several of the severe general side effects could be correlated with traumatic instillation or with instillations given early after the transurethral resection..$^{27,28}$ Therefore, it is recommended not to start the instillations within the first 14 days after the resection or when there is still macroscopic hematuria, suggesting insufficient healing of the resection wound. BCG should not be given after a traumatic catheterization as there is an open wound that can give direct access to the blood circulation provoking disseminated BCG infection. Many experts suggest delaying treatment in the presence of bacterial cystitis because the barrier for the BCG to reach the bloodstream is traumatized. However severe side effects continue to appear even after respecting the aforementioned rules.

\section{Preventive systemic administration of tuberculostatic drugs}

As BCG strains are sensitive for most tuberculostatic drugs, it was logic to explore if its use could diminish the side effects, with however possible less efficacy. EORTC study (30911) addressed this clinical question. Three days of $300 \mathrm{mg}$ isoniazid (or isonicotinylhydrazide [INH]), once daily at the occasion of the BCG instillation, could not reduce local or systemic toxicity ${ }^{29}$ and did not influence efficacy on recurrence and progression. ${ }^{30,31}$ However, INH provoked transient liver toxicity in several patients. So, the use of prophylactic INH is not recommended.

Another prospective, double-blind, placebo-controlled multicenter randomized clinical trial ${ }^{32}$ showed that two doses of $200 \mathrm{mg}$ ofloxacin, which is a strong tuberculostatic agent, given shortly after BCG instillation, reduced moderate-tosevere side effects by $18.5 \%$. Compliance to the therapy was also better. However, it concerned only 115 patients, divided in two groups, which makes definitive conclusions dangerous. As the median follow-up was only 1 year, long-term efficacy cannot be judged. To our knowledge, further reports on this method of prevention did not appear so far, and therefore, it cannot (yet) be advocated.

\section{Preventive symptomatic treatment of bladder irritation during BCG}

As frequency is a major complaint during BCG instillations, $10 \mathrm{mg}$ oxybutynin extended release, twice a day, has been tested in a randomized study versus placebo including 50 patients. ${ }^{33}$ The results were disappointing as the significantly worse outcome was in the oxybutynin arm.

\section{Lowering the BCG dose}

Low-dose BCG has been tried in an attempt to decrease the frequency and severity of the side effects. ${ }^{34}$ In this study, one-third dose BCG was found as effective as a full dose in the prevention of recurrence and progression. However, patients with multifocal tumors fared better with the standard 
dose. In a later study, they confirmed similar efficacy of one-third dose versus full dose in high-grade and high-risk tumors, ${ }^{35}$ while overall side effects were significantly less. However, the number of patients who discontinued BCG for toxicity and the severe complications were similar in both arms. A third report of the same group found that one-sixth dose was significantly less effective than one-third dose, ${ }^{36}$ and therefore should not be used. The EORTC showed in a Phase II marker lesion study that a quarter dose was still effective. ${ }^{37}$ So, this is probably the lowest dose that can be advocated regarding efficacy.

The largest and most recent EORTC study confirms the data on efficacy of the one-third dose BCG but cannot confirm any difference in adverse events with the full dose. ${ }^{9,10}$ The explanation for these differences in outcome between the Spanish and EORTC study is unclear. A major difference in the study design was the duration of BCG treatment, which was 5 months in the Spanish study and 1-3 years in the EORTC study. Anyhow, it is far from evident that a lower dose will diminish side effects in general and certainly not the severe and most troublesome ones, which are the reason for stopping the treatment with BCG.

\section{Use of inactivated BCG}

The idea to inactivate BCG by heat, irradiation, or formaldehyde in order to prevent its side effects due to the living bacteria is already old, but recently was addressed again in preclinical studies. ${ }^{38,39}$ However, one is far from clinical application. Anyhow, one may expect that, if inactivated BCG keeps its therapeutic effect, the side effects due to the immune response will remain.

\section{Treatment of the BCG complications BCG cystitis}

Pollakisuria, dysuria, urgency, and hematuria are frequent in the first 2 days after BCG instillation and do not need therapy. Increased diuresis, to enhance the evacuation of the mycobacteria, is advocated without scientific proof of efficacy, but it seems logic. When side effects remain for longer time or are really intolerable for the patient, symptomatic treatment with spasmolytics, anticholinergics, antiphlogistics, and analgesics are empirically advised. Except for oxybutynin, which failed to be effective, ${ }^{33}$ none of these drugs have been tested in this condition versus placebo. A randomized clinical trial with ofloxacin could diminish local side effects and therefore can be recommended in persisting severe cystitis. ${ }^{32}$ In view of the rather high frequency of associated bacterial cystitis, it is worthwhile to make a culture of the urine and to start thereafter blindly with ofloxacin. This is a pragmatic way of helping a patient who is suffering and claiming therapy. Therapy can be adjusted when the result of the urine culture is available. Anyhow, next instillation should be postponed with at least a week. There is little literature on BCG efficacy altered by changing the intensity of the therapy, but postponing the instillation is the only reasonable solution to keep the patient on further BCG treatment. All these recommendations are based on expert opinion, taken from the previous reviews, as randomized clinical trials or even large observational studies on efficacy of the recommended therapies are missing.

\section{General malaise and fever}

Again, these frequent side effects resolve mostly within 48 hours. Symptomatic antipyretics can be given when fever exceeds $38^{\circ} \mathrm{C}$. Fever $>38.5^{\circ} \mathrm{C}$ for $>2$ days needs close monitoring of the patient and consultation of infectious disease specialist in order to see if there are other causes of fever. While further diagnostic evaluation, prompt treatment with a minimum of two or more tuberculostatic agents (eg, fluoroquinolones, INH, rifampicin) is started. ${ }^{4}$ The duration of this therapy is badly defined and depends on further evolution of fever, malaise, and other findings at diagnostic exploration. Anyhow, further BCG is at least postponed, but with a high-risk tumor, the side effects should be weighed against the benefits.

\section{Local infections with BCG}

BCG can invade the prostate and the seminal vesicles up to the epididymis. These diseases require a triple tuberculostatic treatment. Often fluoroquinolone is one arm of it. The duration varies from one study to another from 3 to 6 months. Orchiepididymectomy may be a rapid solution for a severely symptomatic patient, but it does not replace the general tuberculostatic treatment that should be continued. ${ }^{13}$ In rare cases, transurethral resection of the prostate is necessary to solve obstruction. Asymptomatic granulomatous prostatitis, however, was found to be frequent after intravesical BCG therapy but does not require treatment. ${ }^{14}$

BCG balanitis or contact dermatitis has been described and can be handled with local steroids. Careful cleaning of hands and genital region after drug handling and voiding should prevent it.

Granulomatous balanitis, however, can present with multiple erythematous and painless nodules of the glans, which should be treated with systemic triple tuberculostatic drug therapy. ${ }^{13,40}$ 


\section{Systemic BCG infections}

As described earlier, BCG can spread from the bladder in any organ in the body and provoke a wide range of manifestations, shortly or several months after BCG instillation. All these conditions require cessation of $\mathrm{BCG}$, if still applicable, and a systemic treatment with at least three tuberculostatic agents. The duration of the treatment reported in the literature is variable from 3 months to 1 year, depending on the severity of the complication treated. This is based rather on expert opinion than on any evidence. In general, a 6-month therapy is advocated.

In case of septicemia and multiorgan failure, early highdose corticosteroids support is an essential part of the treatment as long as symptoms persist. In these cases, it is also recommended to start as many as four tuberculostatic agents in order to obtain rapid and trustable response. ${ }^{13}$

Inadvertent intravenous BCG administration was also reported..$^{41}$ This is due to the dubious instruction from the urologist who wrote "IV BCG" (meaning intravesical) for an unexperienced nurse.

\section{Tuberculostatic agents}

The tuberculostatic drugs that can be used against BCG are INH, rifampicin, ethambutol, fluoroquinolones, clarithromycin, aminoglycosides, and doxycycline..$^{42} \mathrm{BCG}$ strains are not sensitive to pyrazinamide, and therefore should not be used, although mentioned in several articles.

The use of cycloserine is controversial. Although it has some survival benefits in mice experiments, the drug seems to be inactive against the current strains of BCG. ${ }^{42}$ Its use cannot be recommended.

Monotherapy is never indicated as resistance to one drug rapidly appears. Triple drugs, and even four drugs in the severe cases, should be used. The drugs are given as a single daily dose, all together before breakfast, on an empty stomach.

Tolerability and side effects may be a serious problem. INH has a well-known liver toxicity, which appears in $10 \%-20 \%$ of the patients. ${ }^{6}$ When liver transaminases exceed three to five times the normal value, the drug must be stopped. It is also recommendable to control the transaminases before start of the treatment. Rifampicin can add to the liver toxicity and has many interactions with other drugs. Ethambutol can provoke optical neuritis. When these drugs are prescribed by physicians who are not familiar with those drugs, it is advocated to consult the professional websites on that subject or to refer patients to colleagues or centers familiar with the treatment of tuberculosis.

\section{Immunological complications}

BCG incites a strong immune reaction, and therefore, immunologically induced diseases and symptoms may be expected. More than 89 cases of arthritis and arthralgia provoked by BCG have been described in a recent review article, ${ }^{43}$ and a more recent case can be added. ${ }^{15}$ Polyarthritis is the most frequent form of the disease, but one or few articulations can be attacked. Mild and short arthralgias, without consequences, occurred regularly during a BCG course. Reiter's syndrome and conjunctivitis alone have been described in association with the arthritis. ${ }^{44}$ Immunological reactions at any other organ in the body theoretically can be expected and are rarely described. ${ }^{13,23}$ The diagnostic workup of these patients may be exhaustive given the broad range of causes of similar symptoms.

Nonsteroidal anti-inflammatory drugs and corticosteroids are effective in the large majority of the cases with recovery within 2 and 6 months, respectively, of $70 \%$ and $93 \%$ of the patients. ${ }^{43}$

\section{Guidelines on nonmuscle-invasive bladder cancer}

BCG is a recommended treatment in all the guidelines on nonmuscle-invasive bladder cancer. In view of the high number of side effects and a small number but severe, potentially lethal complications, it seems reasonable that they give instructions on the management of the complications of BCG. This review draws attention to the fact that some of the suggested methods to prevent BCG toxicity are no longer valid.

An important suggestion is to give written information to patients and their general practitioner on the possible severe, early, and late complications in any organ of the body. Although it has not been proved, it seems a method of common sense to improve early detection and therapy.

\section{Conclusion}

- All physicians giving intravesical BCG should be aware of the possible complications and its treatments.

- The side effects occur from the first instillation to the last one and the incidence is not increasing over time.

- The incidence of bladder irritation, general malaise, and fever is very high, while severe complications remain rare. Following the most recent study, $\sim 8 \%$ of the patients have to stop treatment for these complications.

- BCG infections and reactions can occur at any organ or place in the body, and this can happen shortly or months to years after the BCG treatment, making early diagnosis difficult.

- Additionally, correct diagnosis is hampered by uncertain appearance of BCG in tissues and body fluids. 
- An essential step in the management of BCG complications is written information for the family doctor and for the patient on the possible adverse events and their management.

- Recent data demonstrate that none of the earlier suggested methods to prevent BCG toxicity are valid: lowering the dose, tuberculostatic drugs, or oxybutynin.

- The most common side effects do not need therapy.

- Severe complications are treated with three or four tuberculostatics during 3-12 months depending on the severity of the situation.

- Corticosteroids are an essential therapy in BCG septicemia. Nonsteroidal anti-inflammatory drugs and corticosteroids resolve the immunological complications, mostly rheumatological, of BCG.

\section{Disclosure}

The authors report no conflicts of interest in this work.

\section{References}

1. Redelman-Sidi G, Glickman MS, Bochner BH. The mechanism of action of BCG therapy for bladder cancer - a current perspective. Nat Rev Urol. 2014;11(3):153-162.

2. Brausi M, Witjes JA, Lamm D, et al. A review of current guidelines and best practice recommendations for the management of nonmuscle invasive bladder cancer by the International Bladder Cancer Group. J Urol. 2011;186(6):2158-2167.

3. Burger M, Oosterlinck W, Konety B, et al; International Consultation on Urologic Disease-European Association of Urology Consultation on Bladder Cancer 2012. ICUD-EAU International Consultation on Bladder Cancer 2012: non-muscle-invasive urothelial carcinoma of the bladder. Eur Urol. 2013;63(1):36-44.

4. Alfred Witjes, J, Palou J, Soloway M, Lammd D, Brausie M, Roan Spermona J. Clinical practice recommendations for the prevention and management of intravesical therapy-associated adverse events. Eur Urol Suppl. 2008;7(10):667-674.

5. Colombel M, Picard A. [Prevention of bacillus Calmette-Guerin immunotherapy complications]. Prog Urol. 2008;18(Suppl 5):S105-S110.

6. Lamm DL, van der Meijden PM, Morales A, et al. Incidence and treatment of complications of bacillus Calmette-Guerin intravesical therapy in superficial bladder cancer. J Urol. 1992;147(3):596-600.

7. Saint F, Irani J, Patard JJ, et al. Tolerability of bacille CalmetteGuerin maintenance therapy for superficial bladder cancer. Urology. 2001;57(5): 883-888.

8. Koya MP, Simon MA, Soloway MS. Complications of intravesical therapy for urothelial cancer of the bladder. $J$ Urol. 2006;175(6):2004-2010.

9. Brausi M, Oddens J, Sylvester R, et al. Side effects of bacillus CalmetteGuerin (BCG) in the treatment of intermediate- and high-risk Ta, T1 papillary carcinoma of the bladder: results of the EORTC genito-urinary cancers group randomised phase 3 study comparing one-third dose with full dose and 1 year with 3 years of maintenance BCG. Eur Urol. 2014;65(1):69-76.

10. Oddens J, Brausi M, Sylvester R, et al. Final results of an EORTC-GU cancers group randomized study of maintenance bacillus CalmetteGuerin in intermediate- and high-risk Ta, T1 papillary carcinoma of the urinary bladder: one-third dose versus full dose and 1 year versus 3 years of maintenance. Eur Urol. 2013;63(3):462-472.

11. Brosman SA. The use of bacillus Calmette-Guerin in the therapy of bladder carcinoma in situ. J Urol. 1985;134(1):36-39.
12. van der Meijden AP, Sylvester RJ, Oosterlinck W, Hoeltl W, Bono AV; Group EG-UTC. Maintenance bacillus Calmette-Guerin for Ta T1 bladder tumors is not associated with increased toxicity: results from a European organisation for research and treatment of cancer genito-urinary group phase III trial. Eur Urol. 2003;44(4):429-434.

13. MacleodLC, NgoTC, Gonzalgo ML. Complications of intravesical bacillus Calmette-Guerin. Can Urol Assoc J. 2014;8(7-8):E540-E544.

14. Balasar M, Doğan M, Kandemir A, et al. Investigation of granulomatous prostatitis incidence following intravesical BCG therapy. Int J Clin Exp Med. 2014;7(6):1554-1557.

15. Ben Abdelghani K, Fazaa A, Souabni L, Zakraoui L. Reactive arthritis induced by intravesical BCG therapy for bladder cancer. BMJ Case Rep. 2014;pii:bcr2013202741.

16. Fournier A, Gouriet F, Fournier PE, et al. A case of infectious endocarditis due to BCG. Int J Infect Dis. 2015;35:27-28.

17. Harbjerg JL, Bjerre CC, Lillebaek T, Weinreich UM. [Pulmonal bacillus Calmette-Guerin infection two years after intravesical bacillus CalmetteGuerin installation]. Ugeskr Laeger. 2014;176(25A):V07120381.

18. Iqbal J, Raja M, Leung J. Peritoneal tuberculosis presenting as recurrent peritonitis secondary to treatment with intravesical bacillus CalmetteGuerin in a patient receiving peritoneal dialysis. Clin Kidney J. 2015;8(1):107-108.

19. Marquez-Batalla S, Fraile-Villarejo E, Belhassen-Garcia M, GutierrezZubiaurre N, Cordero-Sanchez M. Disseminated infection due to Mycobacterium bovis after intravesical BCG instillation. World J Clin Cases. 2014;2(7):301-303.

20. Raffray L, Riviere P, Bonnet H, Duffau P, Longy-Boursier M. [Granulomatous hepatitis revealing a Mycobacterium bovis widespread infection following intravesical BCG therapy]. Rev Med Interne. 2015;36(9):626-630.

21. Thupili CR, Chamarthi SK, Ghosh S. Bacillus Calmette-Guerin (osis). J Urol. 2014;191(6):1876-1877.

22. To U, Kim J, Chia D. Disseminated BCG: complications of intravesical bladder cancer treatment. Case Report Med. 2014;2014:362845.

23. Tobiume M, Shinohara T, Kuno T, et al. BCG-induced pneumonitis with lymphocytic pleurisy in the absence of elevated KL-6. BMC Pulm Med. 2014;14:35.

24. Gonzalez OY, Musher DM, Brar I, et al. Spectrum of bacille CalmetteGuerin (BCG) infection after intravesical BCG immunotherapy. Clin Infect Dis. 2003;36(2):140-148.

25. Sylvester RJ, van der MA, Lamm DL. Intravesical bacillus CalmetteGuerin reduces the risk of progression in patients with superficial bladder cancer: a meta-analysis of the published results of randomized clinical trials. J Urol. 2002;168(5):1964-1970.

26. Rentsch CA, Birkhauser FD, Biot C, et al. Bacillus Calmette-Guerin strain differences have an impact on clinical outcome in bladder cancer immunotherapy. Eur Urol. 2014;66(4):677-688.

27. Debois H, Loupi E, Saliou P, Blangy H, Loeuille D, Gillet P. [Evaluation of tolerance to endovesical BCG treatment in France: analysis of severe adverse effects notified in 3 years]. Prog Urol. 2001;11(3): 458-465.

28. Debois H, Loupi E, Saliou P. [Surveillance of the safety of intravesical BCG therapy in France: quantitative analysis of serious adverse events notified over a period of five years]. Prog Urol. 2002;12(4): 604-608.

29. Vegt PD, van der Meijden AP, Sylvester R, Brausi M, Holtl W, de Balincourt C. Does isoniazid reduce side effects of intravesical bacillus Calmette-Guerin therapy in superficial bladder cancer? Interim results of European organization for research and treatment of cancer protocol 30911. J Urol. 1997;157(4):1246-1249.

30. van der Meijden AP, Brausi M, Zambon V, et al; Members of the EORTC Genito-Urinary Group. Intravesical instillation of epirubicin, bacillus Calmette-Guerin and bacillus Calmette-Guerin plus isoniazid for intermediate and high risk Ta, T1 papillary carcinoma of the bladder: a European organization for research and treatment of cancer genito-urinary group randomized phase III trial. J Urol. 2001;166(2): 476-481. 
31. Sylvester RJ, Brausi MA, Kirkels WJ, et al; EORTC Genito-Urinary Tract Cancer Group. Long-term efficacy results of EORTC genito-urinary group randomized phase 3 study 30911 comparing intravesical instillations of epirubicin, bacillus Calmette-Guerin, and bacillus Calmette-Guerin plus isoniazid in patients with intermediateand high-risk stage Ta T1 urothelial carcinoma of the bladder. Eur Urol. 2010;57(5):766-773.

32. Colombel M, Saint F, Chopin D, Malavaud B, Nicolas L, Rischmann P. The effect of ofloxacin on bacillus calmette-guerin induced toxicity in patients with superficial bladder cancer: results of a randomized, prospective, double-blind, placebo controlled, multicenter study. J Urol. 2006;176(3):935-939.

33. Johnson MH, Nepple KG, Peck V, et al. Randomized controlled trial of oxybutynin extended release versus placebo for urinary symptoms during intravesical bacillus Calmette-Guerin treatment. J Urol. 2013;189(4):1268-1274

34. Martínez-Piñeiro JA, Flores N, Isorna S, et al; For CUETO (Club Urológico Español de Tratamiento Oncológico). Long-term follow-up of a randomized prospective trial comparing a standard $81 \mathrm{mg}$ dose of intravesical bacille Calmette-Guerin with a reduced dose of $27 \mathrm{mg}$ in superficial bladder cancer. BJU Int. 2002;89(7):671-680.

35. Martinez-Pineiro JA, Martinez-Pineiro L, Solsona E, et al. Has a 3-fold decreased dose of bacillus Calmette-Guerin the same efficacy against recurrences and progression of $\mathrm{T} 1 \mathrm{G} 3$ and Tis bladder tumors than the standard dose? Results of a prospective randomized trial. J Urol. 2005;174(4 pt 1):1242-1247.

36. Ojea A, Nogueira JL, Solsona E, et al; CUETO Group (Club Urológico Español De Tratamiento Oncológico). A multicentre, randomised prospective trial comparing three intravesical adjuvant therapies for intermediate-risk superficial bladder cancer: low-dose bacillus Calmette-Guerin (27 mg) versus very low-dose bacillus Calmette-Guerin (13.5 mg) versus mitomycin C. Eur Urol. 2007;52(5):1398-1406.
37. Mack D, Höltl W, Bassi P, et al; European Organization for Research and Treatment of Cancer Genitourinary Group. The ablative effect of quarter dose bacillus Calmette-Guerin on a papillary marker lesion of the bladder. J Urol. 2001;165(2):401-403.

38. Secanella-Fandos S, Noguera-Ortega E, Olivares F, Luquin M, Julian E. Killed but metabolically active Mycobacterium bovis bacillus CalmetteGuerin retains the antitumor ability of live bacillus Calmette-Guerin. J Urol. 2014;191(5):1422-1428.

39. Noguera-Ortega E, Rabanal RM, Secanella-Fandos S, Torrents E, Luquin M, Julian E. Gamma-irradiated Mycobacteria enhance survival in bladder tumor-bearing mice although they are less efficacious than live mycobacteria. J Urol. Epub 2015 Jul 10.

40. Linden-Castro E, Pelayo-Nieto M, Alias-Melgar A. Penile tuberculosis after intravesical bacille Calmette-Guerin immunotherapy. Urology. 2014;84(2):e3

41. Akbulut Z, Canda AE, Atmaca AF, Cimen HI, Hasanoglu C, Balbay MD. BCG sepsis following inadvertent intravenous BCG administration for the treatment of bladder cancer can be effectively cured with anti-tuberculosis medications. N Z Med J. 2010;123(1325):72-77.

42. Durek C, Rusch-Gerdes S, Jocham D, Bohle A. Interference of modern antibacterials with bacillus Calmette-Guerin viability. J Urol. 1999;162(6):1959-1962.

43. Bernini L, Manzini CU, Giuggioli D, Sebastiani M, Ferri C. Reactive arthritis induced by intravesical BCG therapy for bladder cancer: our clinical experience and systematic review of the literature. Autoimmun Rev. 2013;12(12):1150-1159.

44. Tinazzi E, Ficarra V, Simeoni S, Artibani W, Lunardi C. Reactive arthritis following BCG immunotherapy for urinary bladder carcinoma: a systematic review. Rheumatol Int. 2006;26(6):481-488.
Research and Reports in Urology

\section{Publish your work in this journal}

Research and Reports in Urology is an international, peer-reviewed, open access journal publishing original research, reports, editorials, reviews and commentaries on all aspects of adult and pediatric urology in the clinic and laboratory including the following topics: Pathology, pathophysiology of urological disease; Investigation and treatment of

\section{Dovepress}

urological disease; Pharmacology of drugs used for the treatment of urological disease. The manuscript management system is completely online and includes a very quick and fair peer-review system, which is all easy to use. Visit http://www.dovepress.com/testimonials.php to read real quotes from published authors. 